\title{
RESEARCH ON 3D SURFACE MEASUREMENT SYSTEM BASED ON LINE STRUCTURE LIGHT
}

\author{
WuFang $^{1}$, Zhou Yufeng ${ }^{2}$, Li Qing ${ }^{3}$ \\ ${ }^{1}$ Shanghai University of Engineering Science, School of Mechanical Engineering, Shanghai, 201620, China \\ ${ }^{2}$ Shanghai University of Engineering Science, School of Mechanical Engineering, Shanghai, 201620, China \\ ${ }^{3}$ Shanghai University of Engineering Science, School of Mechanical Engineering, Shanghai, 201620, China
}

\begin{abstract}
This paper studied the key technologies of $3 D$ surface measurement system based on linear structured light vision sensor, and effectively achieve the integrity measurement of curved surface. The establishment of the line structure light $3 D$ surface vision measurement system with three coordinate measuring machine for motor drive and control device, effectively with high precision motion measurement machine control characteristics applied to 3D surface scanning measurement. The mathematical model of the measurement system is proposed and its working process is described in detail. Calibration of camera calibration for vision measurement system and visual sensor structural parameters were introduced.
\end{abstract}

Keywords: Three dimensional vision measurement; line structured light; camera calibration; light plane calibration

\section{INTRODUCTION}

In the past half century, with the development of digital image processing technology and computer vision technology, many scholars have applied the two techniques to the three-dimensional data measurement of reverse engineering to form three-dimensional visual measurement technology. Based on this technology, The measurement system is called a three-dimensional vision measurement system. At present, 3D vision measurement technology has been widely applied and development prospects, as a modern reverse engineering technology and the realization of the key, the corresponding technology and systems research and development work not only has high academic value, but also to enhance the design field and The level of the manufacturing industry to play a catalytic role and may make a greater contribution to the successful development of three-dimensional visual measurement system will have a huge social benefits and considerable economic benefits.

\subsection{Three-Dimensional Visual Measurement}

\section{Technology Overview}

The three-dimensional visual measurement is realized by the visual method to obtain the three-dimensional geometric information of the measured object. However, the visual measurement method realized by the ordinary camera's imaging process only completes the transformation from three-dimensional to two-dimensional space, so onedimensional information will be lost. In this condition, simply rely on the camera to obtain two-dimensional images is not able to restore the three-dimensional information objects to provide adequate geometric constraints. The fundamental way to solve this problem is to increase the geometric constraint by introducing or supplementing other tools outside the single camera, and to construct the sufficient conditions to solve the three-dimensional spatial information.

\subsection{Several Kinds of Three-Dimensional Visual}

\section{Measurement Method}

According to the different measurement methods and principles, the existing three-dimensional visual measurement, including structured light measurement, stereo vision measurement, single camera measurement and beam adjustment of several methods[1-3].

\subsubsection{Structured Light Measurement}

Laser has the advantages of good direction, high brightness, the use of lasers to do the light source to produce structured light visual measurement is called structured light visual measurement technology. The research on the threedimensional visual measurement of structured light began in the 1970s and developed from the principle of laser triangulation measurement. It is a kind of visual measurement method widely used in industrial field. It has the advantages of simple structure, , High precision, easy to deal with the light bar image, real-time strong and active control and so on.

By introducing the structural light plane and the transformation matrix between the light plane and the camera coordinate system solved by the pre-calibration technique as complementary constraints to eliminate the ambiguity of the inverse mapping from the two-dimensional image space to the three-dimensional space, a threedimensional measurement relation is established. The main measurement principle is to capture the image of structured light stripe with contour constraint by CCD camera, and use the space geometric transform to obtain the 3D space world coordinates of the light stripe in the image. 
The use of lasers in conjunction with different projection systems can produce a variety of modes of structural light: point structure of light (a point), the line structure of light (single-ray), multi-line structured light (multi-line light) and grid structure of light, Gratings form gratings that encode structured light. At present, the research of structured light 3D vision measurement mainly focuses on structure light projection mode, sub-pixel light stripe center extraction and processing algorithm and visual sensor structural parameter calibration.

\subsubsection{Body Vision Measurement}

Stereoscopic vision measurement is based on the principle of stereoscopic parallax. Two or more cameras are used to acquire the same scene of the same object at the same time. By using the theory of polar line geometry and parallax theory, Dimensional geometric information of the object. Stereoscopic vision includes binocular stereo vision, trinocular stereoscopic vision, and binocular stereoscopic vision, in which binocular stereoscopic vision is the simplest stereoscopic vision measurement model, trinocular stereo vision and multi-eye stereoscopic vision can be seen as binocular stereo vision In the mathematical modeling they are able to binocular stereo vision model-based.

\subsubsection{Camera Measurement}

Single-camera measurement refers to a single camera using a single object to be measured and combined with the control point technology, to achieve the measured object space three-dimensional geometric information method. Since it is not possible to retrieve 3D information from a single 2D image, it is possible to realize the measurement of $3 \mathrm{D}$ geometric information of a space object on the basis of a single image by adding a geometric constraint to the perspective model by using the control point technique.

\subsubsection{Beam Adjustment Measurement}

The beam adjustment measurement is based on the geometric model of the spatial intersection of the imaging beam and the beam adjustment optimization algorithm is the core. The camera is used to set up a plurality of stations at different positions in the measurement space, and the measurement images are acquired from the different pose positions. The high accuracy image processing algorithm and the same name point automatic registration technique are used to obtain the iterative condition of beam adjustment. The beam adjustment algorithm is used to solve the precise $3 \mathrm{D}$ coordinate of the measured point.

\section{STRUCTURED LIGHT 3D SURFACE VISION MEASUREMENT SYSTEM}

Optical triangulation measurement principle is the technical basis of line-structured optical three-dimensional vision measurement system. The principle is to project the linear laser bar to the surface of the measured object, and the light bar is deformed by the modulation of the surface shape of the measured object. By extracting the deformation of the stripe image, by extracting the optical stripe center coordinates on the CCD imaging surface of the offset, you can get the object of a cross-section of two-dimensional data, plus one-dimensional scanning movement can be measured on the surface of the three-dimensional data [4]. Three-dimensional vision measurement system consists of two major components: the visual sensor and motion drive and control equipment. After decades of research and development, the existing measurement system with a variety of different structure of the light projection mode and the number of different combinations of cameras and installation structure, while motion-driven and control equipment also showed a variety of development and appeared to CMM Or joint arm manipulator as the main structural form and data integration as one of the integrated measurement system [5].

\subsection{Measurement System Structure}

In this paper, the structure of the measurement system is composed of a line-structured light vision sensor and a CMM motion and control device, besides the computer equipment. The line laser and the CCD camera of the linestructured light vision sensor are respectively adjusted at a certain angle, and the line laser projects the line structure light to the surface of the measured object. After the modulation of the line-structured light bar of the measured object surface, Of the light stripe image and the image of the imaging interface in the middle position to reduce the distortion caused by the camera error. Line laser and CCD camera were installed in the equipment support frame, the front and rear position can be flexibly adjusted.

CMM movement and control equipment, including control cabinet, control handle, front and rear left and right moving rail, measuring arm composition. Through the measuring arm of the CMM control line-structured optical vision sensor to move forward and backward to complete the scanning measurement tasks. In the CMM equipped with the measurement software can be fixed on the arm in the visual sensor movement position, speed, time and other parameters to set and modify the motion.

\subsection{Measuring System Works}

\subsubsection{Mathematical Model of the Measuring System}

As shown in Figure 1 for the line structure of the threedimensional surface optical measurement system, the mathematical model. According to the measurement method and idea of the system, the following coordinate system is constructed:

Dimensional coordinate system $\mathrm{o}_{\mathrm{s}}-x_{s} y_{s} z_{s}$, also known as the global measurement coordinate system, the final data of the measurement system are converted to the coordinate system, using this data to build the surface of the object measured three-dimensional data profile.

Three-dimensional space Right angle light plane coordinate system $\mathrm{o}_{\mathrm{m}}-x_{m} y_{m} z_{m}-$ also known as light plane 
measurement coordinate system or local measurement coordinate system. The axis of the coordinate system $y_{m}$ is parallel to the axis of the measuring coordinate system $y_{s}$. The axis $x_{m}$ and the axis $z_{m}$ are at an angle $\theta$ from the axis $x_{s}$ and $z_{s}$ can be measured with an angle meter. When the object is measured, the measurement data of the twodimensional coordinate system $\mathrm{o}_{\mathrm{m}}-x_{m} y_{m}$ in the coordinate system will be obtained firstly. Then the data will be transformed into the global measurement coordinate system according to the transformation relation matrix of the two coordinate systems in order to facilitate the subsequent data processing.

Three-dimensional rectangular camera coordinate system $\mathrm{o}_{\mathrm{c}}-x_{c} y_{c} z_{c}$ - in the calibration of the measurement system of the visual sensor structure parameters, the use of the camera coordinate system will be calibrated target on the three-dimensional coordinate system to convert the light plane measurement coordinate system.

Two-dimensional plane orthogonal coordinate system $O_{1}-X Y$ - This coordinate system is the coordinates of the feature points in the image plane, that is, the physical coordinates of the image in the two-dimensional coordinate system.

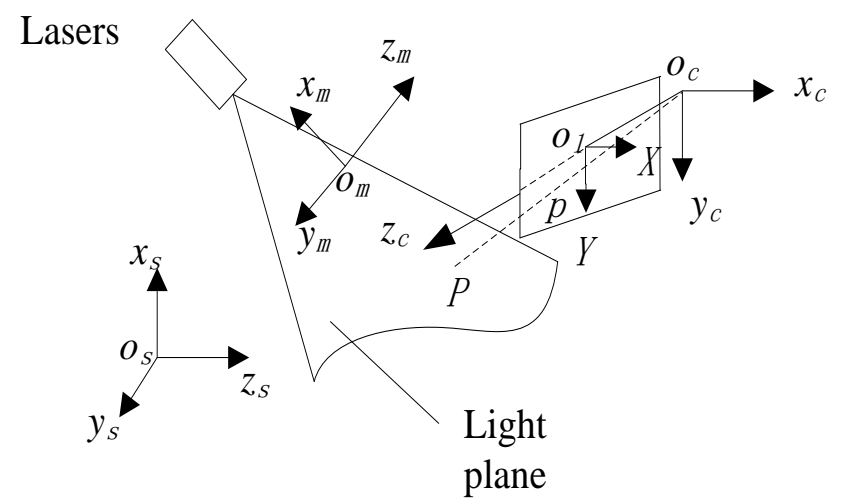

Fig.1 Mathematical model of visual measurement system

\subsubsection{Measuring System Work Process}

Figure 2 shows the measurement system for the work process. First of all, install and connect equipment, debugging the various components of the system, the production of a A4-sized checkerboard calibration paper, and its fixed to a flat rubber plate, placed to fix the measured object, and then follow the steps below.

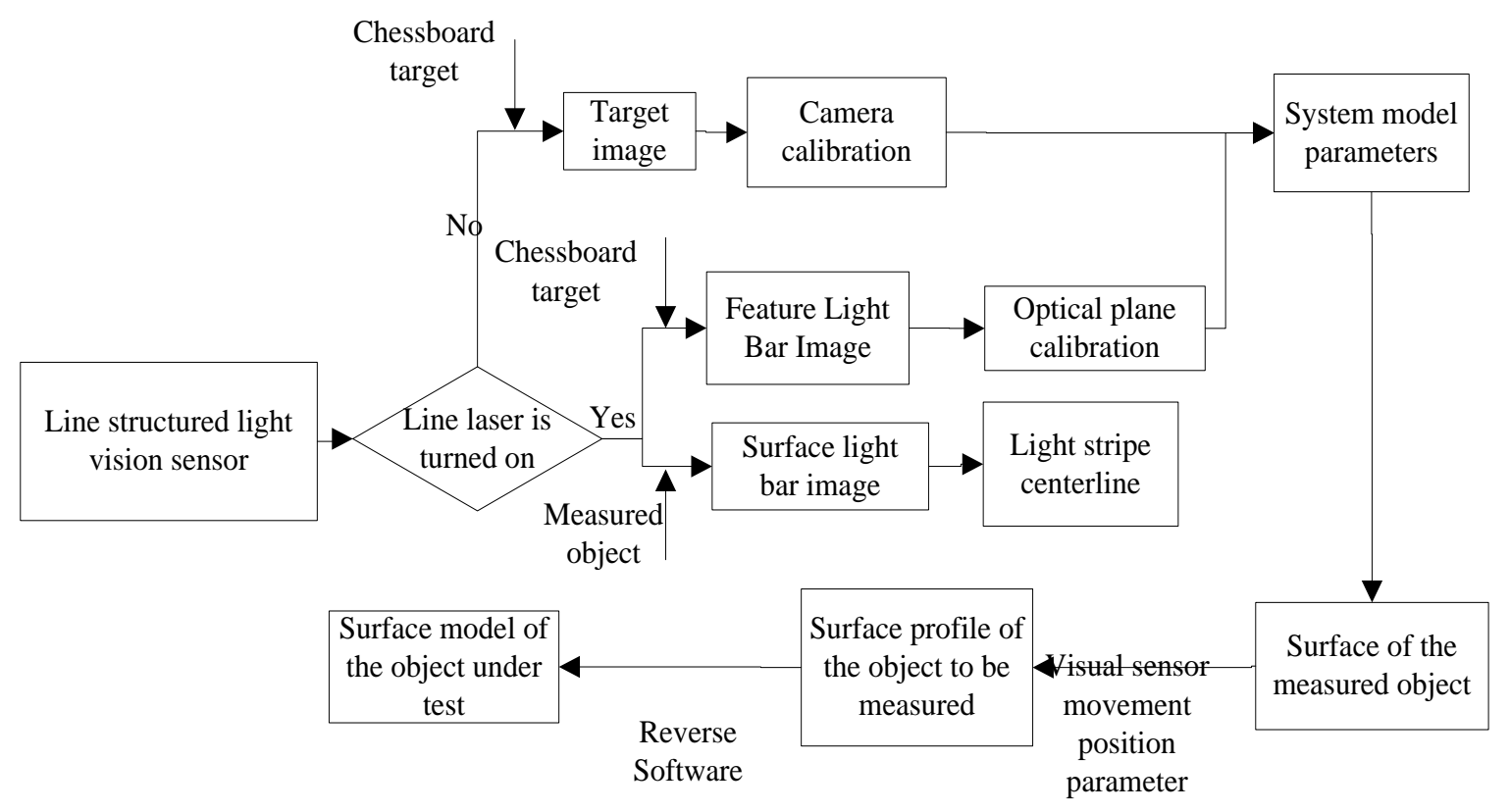

Fig.2 the measurement process

\subsubsection{Camera Calibration}

Line laser without opening, using the image acquisition and processing software, free to move the checkerboard plane calibration plate to a number of different positions, shooting more than 5 checkerboard calibration plate image, the use of nonlinear global optimization Zhang Zhengyou algorithm can calibrate the camera's internal Parameter matrix and two kinds of distortion parameters.

\subsubsection{Light Plane Calibration (Visual Sensor}

\section{Structure Parameter Calibration)}

After obtaining the internal parameter matrix of the camera, the line laser light source is opened, and the light stripe of the line structure is projected onto the checkerboard calibration plate, and three or more positions of the checkerboard are moved freely to obtain three or more light stripe feature line images. Then, the transformation matrix 
of the image plane coordinate to the light plane coordinate is obtained according to the light plane calibration algorithm based on the free moving target of Zhou et al., Which is the structure parameter matrix.

\subsubsection{Light Stripe Center Extraction}

The measured object placed in a position and fixed to the table, adjust the placement angle and try to avoid blocking light bar phenomenon. Measure software PC through CMM. DIMS sets the motion control position and other related parameters, at a fixed interval of different locations to shoot a group of linear motion scanning light stripe image, and then use the paper to achieve the optical stripe center extraction algorithm for each image of the optical stripe centerline and coordinates point.

\subsubsection{Measured Object Surface Profile}

The light stripe centerline of each light stripe image of the captured image group is combined with the motion control position parameter and the light plane coordinate to the global coordinate transformation matrix so as to align the center line of each light stripe in a one-dimensional scanning manner in a specific Position, forming the measured object surface contour.

\subsubsection{Surface Reverse Modeling}

The surface contour data obtained in the above steps are introduced into the reverse engineering design software, and the surface reverse modeling can be done by using these point cloud data to obtain the surface model of the measured object.

\section{PARAMETER CALIBRATION OF VISUAL MEASUREMENT SYSTEM}

In the vision measurement system, to realize the transformation of the two-dimensional image coordinate to the light plane measurement coordinate or the global measurement coordinate, it is necessary to know the system model parameters, which are obtained by the calibration experiment. The calibration of system model parameters mainly refers to the parameter calibration of line-structured light vision sensor. The parameters of the line-structured light vision sensor include the internal parameters (focal length, principal point and distortion coefficient) of the camera and the structural parameters of the visual sensor (the position and orientation of the camera coordinate system relative to the optical plane coordinate system). Therefore, the measurement system model parameter calibration will be divided into camera internal parameter calibration and visual sensor structure parameter calibration (light plane calibration) two parts.

\subsection{Camera Internal Parameter Calibration}

In the vision-based measurement and detection system, the camera must be parameter calibration, which is based on visual automation of the basic work. The performance of the whole visual system depends mainly on the calibration accuracy of the camera parameters, especially for the application of three-dimensional measurement, the calibration results will directly affect the accuracy of the measurement results of the whole system [6]. The camera calibration process is to establish the relationship between the camera image coordinates and the world coordinates of the measured object, the way is based on the camera mathematical model, from the known feature point image coordinates and object world coordinates conversion relationship, coordinate transformation and optimization algorithm In order to achieve the model parameters of the camera [2,7].

\subsection{Visual Sensor Structure Parameter Calibration}

\section{[8]}

According to the surface model of the line-structured light vision measurement system, the calibration of the linestructured optical vision sensor mainly refers to the determination of the structural parameters of the visual sensor after the calibration of the internal parameters of the camera, mainly the corresponding transformation relationship between the light plane and the twodimensional image plane. Its structural parameter transformation matrix.

\section{CONCLUSION}

With the development of image engineering and computer vision technology, 3D vision measurement technology has been extensively researched and applied in reverse engineering. It has played a great advantage in professional measurement and detection and engineering application. On the basis of summarizing the characteristics of existing visual measurement methods and their measurement system characteristics, in order to achieve the integrity of the surface of the surface and high precision measurement requirements, this paper based on line-structured optical vision sensor 3D surface measurement system key technology And the surface integrity measurement of the surface is realized effectively.

\section{ACKNOWLEDGMENT}

The authors acknowledge Shanghai University of Engineering and Science for giving financial supports to this project with No. 16KY0108.

\section{REFERENCES}

[1]. Zheng Jigui, Yu Zhijing. Visual measurement principle and method [M]. Beijing: Mechanical Industry Press, 2012.

[2]. Zhang Guangjun. Machine Vision [M]. Beijing: Science Press, 2010.

[3]. Sun Changku, Ye Shenghua. Laser measurement technology [M]. Tianjin: Tianjin University Press, 2015.

[4]. Liu Zhigang. Research on CMM Integrated Measurement Technology of Reverse Engineering Midline Structure Light Vision Sensor [D]. Xi'an Jiaotong University, 2014. 
[5]. Lin Na. Study on three - dimensional laser vision measurement and its calibration technology [D]. Dalian Maritime University, 2007.

[6]. Wuhan University Digital Photogrammetry and Computer Vision Research Center website: www. Whudpcv. Cn / Research. Asp? LD = 318 \& www. Whudcv. Cn / Research. Asp? ID = 317 .

[7]. Zhou Fuqiang. Research on the key technology of binocular stereo vision detection [R]. Beijing University of Aeronautics and Astronautics, 2012.

[8]. D. Bhatnagar, eta1. Static scene. E analysis using structured light. Image and Vision Computing, 2013. 